\title{
Comprehensive analysis of metastasis-related genes reveals a gene signature predicting the survival of colon cancer patients
}

\author{
Haotang Wei ${ }^{1}{ }^{,}$Jilin Li $^{2}{ }^{2}$ Minzhi Xie ${ }^{2}$, Ronger Lei ${ }^{3}$, Bangli Hu ${ }^{\text {Corresp. }} 2$ \\ 1 Department of Gastrointestinal Surgery, Third Affiliated Hospital of Guangxi Medical University, Nanning, China \\ 2 Department of Research, Affiliated Tumor Hospital of Guangxi Medical University, Nanning, China \\ 3 Department of Gastroenterology, First Affiliated Hospital of Guangxi Medical University, Nanning, China \\ Corresponding Author: Bangli $\mathrm{Hu}$ \\ Email address: hubangli@gxmu.edu.cn
}

Objective The mechanism underlying colon cancer metastasis remain unclear. This study aimed to elucidate the genes alteration during the metastasis of colon cancer and identify genes that crucial to the metastasis and survival of colon cancer patients. Methods The dataset of primary and metastasis tissue of colon cancer, and dataset of high and low metastasis capability of colon cancer cells were selected as training cohort, and the overlapped differentially expressed genes (DEGs) were screened from the training cohort. The functional enrichment analysis for the overlapped DEGs was performed. The prognostic value of overlapped DEGs were analyzed in TCGA dataset, and a gene signature was developed using genes that related to the overall survival (OS). The prognostic value of the gene signature was further confirmed in a validation cohort. Results 184 overlapped DEGs were screened from the training cohort. Functional enrichment analysis revealed the significant gene functions and pathways of the overlapped DEGs. Four hub genes (OXCT1, ACTN4, IL-8, ITGA3) were identified using protein-protein network analysis. Six genes (ALDH2, NEDD9, FLNA, LBR, TWF1, SRSF1) were closely related to the OS of colon cancer patients. A gene signature was developed used these six genes based on their risk score, and the validation cohort indicated that the prognostic value of this gene signature was high in the prediction of colon cancer patients. Conclusions Our study demonstrates a gene profiles related to the metastasis of colon cancer, and identify a sixgene signature that acts as an independent biomarker on the prognosis of colon cancer. 
1

2 Comprehensive analysis of metastasis-related genes reveals a gene signature predicting the

5 Haotang Wei ${ }^{1 \#}, \quad$ Jilin Li ${ }^{2 \#}, \quad$ Minzhi Xie ${ }^{2}, \quad$ Ronger Lei ${ }^{3}, \quad$ Bangli Hu ${ }^{2}$

61 Department of Gastrointestinal Surgery, the Third Affiliated Hospital of Guangxi Medical

7 University, Nanning 530031, China

82 Department of Gastroenterology, the First Affiliated Hospital of Guangxi Medical University,

$9 \quad$ Nanning 530021, China

103 Department of Research, Affiliated Tumor Hospital of Guangxi Medical University, Nanning 11 530021, China.

12 \#These authors contributed equally to this work.

14 Corresponding author: Bang-li $\mathrm{Hu}$

Department of Research,

16 Affiliated Tumor Hospital of Guangxi Medical University,

1771 Hedi Road, Nanning 530021, China

18 Tel: +86-0771- 5321865; Fax +86-0771- 5321865

19 E-mail: gxhubl@163.com 


\section{Abstract}

Objective The mechanism underlying colon cancer metastasis remains unclear. This study aimed to elucidate the gene alterations during metastasis of colon cancer and identify genes crucial to understanding the metastasis and survival of colon cancer patients. Methods The dataset of primary and metastatic tissue of colon cancer and the dataset of high and low metastatic capability of colon cancer cells were selected as a training cohort, and the overlapped differentially expressed genes (DEGs) were screened from the training cohort. Functional enrichment analysis for the overlapped DEGs was performed. The prognostic value of overlapped DEGs was analyzed in the TCGA dataset, and a gene signature was developed using genes that related to the overall survival (OS). The prognostic value of the gene signature was further confirmed in a validation cohort. Results A total of 184 overlapped DEGs were screened from the training cohort. Functional enrichment analysis revealed significant gene functions and pathways of the overlapped DEGs. Four hub genes (OXCT1, ACTN4, IL-8, ITGA3) were identified using protein-protein network analysis. Six genes (ALDH2, NEDD9, FLNA, LBR, TWF1, SRSF1) were closely related to the OS of colon cancer patients. A gene signature was developed using these six genes based on their risk score. The validation cohort indicated that the value of this gene signature was high in the prognostic prediction of colon cancer patients. Conclusions Our study demonstrates gene profiles related to the metastasis of colon cancer and identifies a six-gene signature that acts as an independent biomarker for the prognosis of colon cancer. 


\section{Introduction}

Colorectal cancer $(\mathrm{CRC})$ is one of the leading malignant cancers in the world, and colon cancer accounts for a large part of CRC (Siegel et al. 2017a; Siegel et al. 2017b). During the last three decades, with the development of new therapies, such as asbevacizumab and anti-epidermal growth factor receptor antibody (Castro et al. 2013; Knijn et al. 2010), survival outcomes for colon cancer patients with localized- and regional disease have improved greatly. However, cancer metastasis remains one of the main causes of death in these patients. One studied showed that the prognosis of patients with distant metastasis remained poor compared to the prognosis of patients with early-stage colon cancer (Siegel et al. 2017b). Accumulation of genetic mutations is recognized as one of the important causes for the pathogenesis and progression of colon cancer (Sameer 2013). Hence, exploring the mechanisms of cancer metastasis and searching suitable predictors are crucial to the diagnosis and treatment of colon cancer.

Previously, the TNM stage and pathological characteristics of colon cancer have been used to predict the prognosis and facilitate treatment for colon cancer patients. However, there are some limitations in using these methods to assess patients (Marzouk \& Schofield 2011). Recently, several novel biomarkers have been tested with the aim of improving the prediction of therapeutic response and prognosis of colon cancer patients (Demirkol et al. 2017; Hu et al. 2014; $\mathrm{Xu}$ et al. 2017). These have aided in the diagnosis and treatment of colon cancer, but the results were inconsistent and need to be further studied.

Thus far, metastasis is a major factor for poor prognoses of colon cancer patients, and the liver is the most common organ affected. However, the molecular mechanism underlying distant metastasis remains unclear. Therefore, a comprehensive analysis of the molecular alterations to identify prognostic indicators is key for the management of colon cancer patients with distant 
66 metastasis. In this study, by using the colon cancer data from gene expression omnibus (GEO)

67 and The Cancer Genome Atlas (TCGA), we analyzed the data of patients with colon cancer

68 using primary tumor samples and liver metastasis samples to unveil the genes key to the

69 development colon cancer metastasis and the potential prognostic indicators.

\section{Materials and methods}

72

73

\section{Patient datasets}

The colon cancer tissue and cells microarray data (GSE40367(Roessler et al. 2015) and GSE2509 (Provenzani et al. 2006)) were retrieved and downloaded from the GEO (http://www.ncbi.nlm.nih.gov/geo/) database of National Center for Biotechnology Information (NCBI) as the training cohort. The GSE40367 dataset includes seven colon adenocarcinoma (COAD) with liver metastasis species and eight COAD primary tumor species. The GSE2905 dataset includes two colon cancer cell lines (SW480: low metastasis capability and SW620: high metastasis capability). The prognostic value of genes was analyzed using the data of COAD from TCGA. To confirm the results from training cohort, we used the GSE41258 (Sheffer et al. 2009) dataset that includes 390 species as a validation cohort. Because the data were download freely from GEO and TCGA database, approval of the ethics committee of Guangxi Medical University was not needed.

\section{Identification of overlapped DEGs}

R language software (version 3.4.2) and Bioconductor packages were applied to screen the differentially expressed genes (DEGs) between primary tumor tissue and liver metastasis tissue in GSE40367. The DEGs between SW480 and SW620 in GSE2905 were also screened. Genes 
89

90

91

92

93

94

95

96

97

98

99

100

101

102

103

104

105

106

107

108

109

110

111

112

113

114

115

116

that fulfilled the criteria of having a $\mathrm{p}$ value $<0.05$ and $|\operatorname{logFC}| \geq 1$ were defined as the DEGs. Then, the intersected DEGs of GSE40367 and GSE2905 were defined as overlapped DEGs. The probe level GSE data were converted into gene expression values to measure each gene before the screening of DEGs. If one gene corresponded to multiple probe sets, we used the average data of the multiple probes as the gene expression values (Qin et al. 2012). We also eliminated genes that had over $>20 \%$ values of the total samples as a previous study did (Liew et al. 2011). After pre-processing the data, t-test methods were used to screen the DEGs using a limma package.

\section{Functional enrichment analysis of overlapped DEGs}

Gene Ontology (GO) includes three categories, namely, the biological process (BP), molecular function (MF), and the cellular component (CC). To investigate the functional level of DEGs, the genes underwent GO analysis using the Database for Annotation, Visualization and Integrated Discovery (DAVID, https://david.ncifcrf.gov/). The significant GO categories were defined as those with $\mathrm{p}<0.05$. Following this, KEGG pathway analysis was conducted to identify significant pathways with gene enrichment using a DAVID online tool. We defined pathways as significant if the $\mathrm{p}$ value was $<0.05$.

\section{Integration of PPI network and subnetwork analysis}

Protein-protein interaction (PPI) networks was used to identity key genes and important gene modules which are involved in cancer development by assessing gene interaction levels. In this study, the data of PPI network for overlapped DEGs was acquired from the Search Tool for the Retrieval of Interacting Genes (STRING) database (http://www.string- db.org/). Then, Cytoscape software (version 3.5.2) was used for the establishment of a PPI network for all overlapped DEGs. After the establishment of a PPI network, module analysis was carried out by Molecular Complex Detection to detect the gene modules of the PPI network. The hub of genes of each module was identified based on the score of each gene in the module. 


\section{Acquisition of a gene signature from the training cohort}

118 The association of overlapping DEGs with the overall survival (OS) of colon cancer patients

119

120

121

122

123

124

125

126

127

128

129

130

131

132

133

134

135

136

137

138

139

140

141

142

143

144

145

146

147

was analyzed in the COAD dataset from the TCGA database using a Cox regression analysis in the survival package of $\mathrm{R}$. The gene with $\mathrm{p}<0.05$ was considered to be an independent prognostic factor. To estimate the relative contribution of multiple genes for survival prediction of colon cancer patients, the hub genes were applied to develop a prognostic model using the risk scoring system. In brief, the risk score system was calculated based on a linear combination of the expression level multiplied by the regression coefficient derived from the multivariate cox regression model (the beta value) with the following formula as previously reported: Risk score $=$ expression of Gene ${ }_{1} \times \beta_{1}$ Gene $_{1}+$ expression of Gene $2 \times \beta_{2}$ Gene $_{2}+\ldots$ expression of Gene $\times$ $\beta_{\mathrm{n}}$ Gene $_{\mathrm{n}}{ }^{[12]}$. Using the median risk score as the cutoff, patients were divided into high-risk and low-risk groups. Kaplan-Meier curves were used to estimate the difference in survival for patients between the high-risk group and low-risk groups. $\mathrm{p}<0.05$ was defined as significantly different. Time-dependent receiver operating characteristic (ROC) curve analysis for the gene signature was preformed using the R package "survivalROC" (Heagerty et al. 2000). All statistical analyses were performed using R software and Bioconductor.

\section{Results}

Overlapped genes of CRC cells and tissues

The DEGs of GES2905 and GSE40367 were screened on the basis of the selection criteria after preprocessing the raw data. A total of 341 DEGs between colon cancer cells lines SW420 and SW680 cells were identified in GES2905 dataset, and 7339 DEGs between primary and metastasis tumor specimens were identified in the GSE40367 dataset. By overlapping the DEGs from the two datasets, we obtained 184 overlapped genes that differentially expressed in both CRC cells and tissues. The result is shown in Fig. 1.

\section{GO and KEGG enrichment analysis}

The gene functions of the 184 overlapped DEGs were then analyzed by GO and KEGG enrichment analysis. Using the DAVID online tools, we found that the most enriched GO terms of DEGs related to BP were Signal transduction, and the MF was Protein binding, and the $\mathrm{CC}$ was Cytoplasm. The KEGG pathway analysis based on the GO results revealed that Thyroid 
148 hormone synthesis was the most significant pathway of the overlapped DEGs. The results are 149 shown in Fig. 2.

150

151

152

153

154

155

156

157

158

159

160

161

162

163

164

165

166

167

168

169

170

171

172

173

174

175

176

177

178

\section{PPI network and Module screening analysis}

Using data from the STRING database, a PPI network for the 184 DEGs consisting of 133 nodes and 138 edges was constructed using Cytoscape software. The overall PPI network is shown in Fig. 3A. Following this, the plug-in MCODE was used to detect the modules in the network, and four modules from the PPI network were identified, with the OXCT1 (sore: 4), ACTN4 (sore: 3), IL-8 (sore: 3), ITGA3 (sore: 3) as the hub genes of each module. The top three modules are shown in Fig. 3B-D.

\section{Prognostic value of overlapped DEGs}

The prognostic value of the 184 overlapping DEGs was analyzed using the COAD dataset of TCGA by multiple Cox regression analysis after adjusting the data for age, sex, and TNM stage. The results showed that only $A L D H 2$, NEDD9, FLNA, LBR, TWF1 and SRSF1 were independent genes that associated with the OS of colon cancer patients, with beta values of $-1.343,-0.051$, $0.492,-0.020,-0.181$ and -1.938 , respectively. We developed a six-gene signature by calculating the risk score of each gene, and divided the patients into a high-risk group and a low-risk group on the basis of the median risk score (Fig. 4A), the survival status, and the genes expression level shown in Fig. 4B-C. The survival analysis revealed that this six-gene signature within the highrisk group predicted poor OS of colon cancer patients compared to that in the low risk group, with $\mathrm{p}<0.001$ (Fig. 5A). Using survival ROC analysis, we found that the risk score of this sixgene signature could moderately predict the 1-, 3-, 5-year OS of colon cancer patients, as the value of the area under ROC curve (AUC) was 0.686, 634, and 618, respectively (Fig. 5B).

\section{Validation cohort confirms the prognostic value of the six-gene signature}

The prognostic value of the six-gene signature for the OS of colon patients was further determined in the validation cohort (GSE41258 datasets, 390 colon patients, mean follow-up 65.3 months). By using the same risk score model and cutoff value derived from the training cohort, 390 patients of the validation cohort were classified into either a high-risk group $(n=195)$ or a low-risk group $(n=195)$. Consistent with results of the training cohort, the results of this six- 
179

gene signature indicated an obvious difference between the high risk-group and low-risk group with regard to the OS of colon cancer patients ( $p=0.005$, log-rank test)

\section{Discussion}

Metastasis accounts for $90 \%$ of the mortalities of colon cancer patients and thus has become the most lethal characteristic of colon cancer ( $\mathrm{Li}$ et al. 2017). Colon cancer patients with localized and regional disease have a good prognosis (the 5-year survival rate is up to 91.1\%), but patients with distant metastasis have a much worse prognosis (the 5-year survival rate has dropped to 13.3\%) (Siegel et al. 2017a). Furthermore, the failure of treatment is mostly caused by the metastatic dissemination of primary tumors (Deliu et al. 2014; Stein \& Schlag 2007). At a molecular level, distinct metastasis of colon cancer is molecularly and clinically distinct from the primary site of origin (Zarour et al. 2017). Thus, analyzing the molecular alteration of colon cancer with distinct metastasis is beneficial for the identification of candidate targets of early diagnosis and treatment of advanced stage colon cancer.

To date, some biomarkers have been identified as candidate targets for early diagnosis and treatment of colon cancer and rectal cancer, including genes, miRNA, lncRNA and the related signatures. Some gene signatures related to the metastasis potential of each tumor have been described, with promising results. Vellinga et al (2017) designed a lymphangiogenic gene set, applied it to large datasets of $\mathrm{CRC}$, and found that this lymphangiogenic gene set was related to a worse prognosis. Rokavec et al (2017) reported a single gene, RBM47, down-regulated during CRC progression may promote epithelial-mesenchymal transition and metastasis. Furthermore, proteomic studies used exosomes that from cancer cell lines and identified four candidate genes (MET, S100A8, S100A9, TNC) associated with CRC metastasis (Ji et al. 2013). Other biomarkers, such as a four-miRNA signature (let-7i, miR-10b, miR-221, and miR-320a) (Hur et 
203 al. 2015), and a six-lncRNA signature were reported to be promising biomarkers for the 204 metastasis and prognosis of CRC (Hu et al. 2014).

205 In this study, we identified 4 hub genes from the subnetwork of the PPI network: OXCT1, 206 ACTN4, IL-8 and ITGA3. OXCT1 is a key enzyme involved in the process of ketone body 207 metabolism, and catalyzes the first and rate-determining step of ketolysis. OXCT1 can be 208 converted into acetyl-CoA during the process of metabolism and takes part in the tricarboxylic 209 acid cycle for the oxidation and the production of ATP (Zhang \& Xie 2017). The role of 210 OXCT1 has been implicated in several cancers, included colorectal cancer, and OXCT1 was 211 overexpressed in the metastatic CRC cell line CC-M3 (Lee et al. 2016). ACTN4 is a non-muscle212 type alpha-actinin, which plays an important role in regulating cytoskeleton organization and is 213 involved in transcriptional regulation of gene expression. ACTN4 encodes a nonmuscle, alpha214 actinin isoform that is concentrated in the cytoplasm; participates in metastatic processes; and 215 facilitates the motility, invasion, and metastasis of cancer cells. In CRC, ACTN4 was reported to 216 promote CRC cell line invasion by suppressing focal adhesion maturation (Fukumoto et al. 2015). 217 ITGA3 belongs to the integrin family, joins a beta 1 subunit to form an intact integrin and 218 interacts with several extracellular matrix proteins (Nagata et al. 2013). ITGA3 was found to be

219

220

221

222

224 225 associated with lymphatic dissemination and local invasiveness in cancers. One study showed that ITGA3 was over-expressed in stages III versus I of CRC patients and is related to OS and disease-free survival (Linhares et al. 2015). IL-8 is an important pro-inflammatory chemokine and plays a role in the recruitment of leukocytes to the sites of infection or tissue injury. Growing evidence suggests that paracrine signaling by tumor-derived IL-8 promotes the trafficking of neutrophils and myeloid-derived suppressor cells into the tumor microenvironment, which is associated with dampened anti-tumor immune responses (David et al. 2016). Lambrechts et al 
226 (Lambrechts et al. 2015) observed that IL-8 plasma levels at baseline and subsequent increases in

227 IL-8 were associated with worse progression-free survival in metastatic CRC patients. These

228 studies confirmed our results that the hub genes of the PPI network were crucial to the metastasis

229 of colon cancer, but further studies are warranted to determine the underlying mechanism.

230 Similar to the previous studies, in this study, a signature constructed by six genes was shown

231 to be a good predictor of the OS of patients with colon cancer. Among these six genes, the

232 ALDH2, NEDD9, SRSF1, and FLNA were reported to be associated with the CRC in several

233 studies. ALDH2 is essential for the metabolism and detoxification of a wide range of

234 endogenous and exogenous aldehyde substrates. ALDH2 is the rate-limiting enzyme in the

235 ethanol metabolism, oxidizing acetaldehyde to acetic acid both in the liver and other tissues

236 (Chen et al. 2016). As a novel biological marker, ALDH2 is an attractive prospect in the

237 screening, diagnosis, and evaluation of the prognosis of many diseases, and the genetic

238 polymorphism of ALDH2 significantly correlated with the susceptibility to CRC (Li et al. 2016).

239 NEDD9 is a non-catalytic scaffolding protein that assembles complexes involving oncogenic

240 kinases and regulates the magnitude and duration of cell signaling cascades that control multiple

241 processes important to the development and progression of tumors (Shagisultanova et al. 2015).

242 Studies have shown that downregulation of NEDD9 by apigenin can suppresses migration,

243 invasion, and metastasis of CRC cells (Dai et al. 2016). With regard to SRSF1, one study

244 reported that phosphorylation of SRSF1 regulated alternative splicing of tumor-related Rac1b in

245 CRC cells (Goncalves et al. 2014). SRSF1 is classified as an exonic splicing enhancer and

246 recognizes degenerate purine-rich sequence motifs. In addition, it can promote the recognition of

247 both constitutive and alternative exons during the process of spliceosomal assembly (Sanford et

248 al. 2009). FLNA is an actin-binding protein expressed ubiquitously in the body, involved in 
249 many cell-signaling pathways, and it is important in the maintenance of cell shape and motility.

250 The mutation of FLNA has been shown to be associated with the neuronal migration, vascular

251 function, connective tissue integrity, and skeletal development (Shelmerdine et al. 2017); FLNA

252 showed low expression in CRC patients and was significantly correlated with the incidence and 253 development of CRC (Tian et al. 2015). LBR, a transmembrane protein of the inner nuclear 254 membrane, interacts with heterochromatin and B-type lamins through its nucleoplasmic amino255 terminal domain and is phosphorylated throughout the cell cycle (Duband-Goulet et al. 1998). 256 TWF1 belongs to the ADF-H family and is a conserved actin-binding protein. TWF1 regulates 257 diverse morphological processes through sequestering ADP-actin monomers or capping filament 258 barbed ends (Paavilainen et al. 2007). However, no study has yet reported the role of LBR and 259 TWF1 in CRC and therefore their role needs be investigated further in colon cancers.

260 Compared with previous studies, this study screened the metastasis-related genes by 261 overlapping the DEGs from cancer tissues and cell lines. These tissues included primary colon 262 cancer tissues and liver metastasis tissues, and the cell lines included primary (SW480) and 263 metastatic (SW620) human isogenic colorectal cancer cell lines. Thus, the overlapped DEGs 264 could more reliably reflect the gene alteration in metastatic colon cancer. Some limitations of this study need to be noted. First, although metastasis-related genes of colon cancer were identified and their prognostic value was validated in our study, the results were calculated from microarray or RNA-sequencing technique datasets and thus lack of functional validation of the target genes is one of the major limitations of this study. Therefore, thorough functional experiments for these genes and corresponding downstream events to reveal novel diagnostic and therapeutic targets for colon cancer is necessary. Second, the development of colon cancer metastasis can be caused by many factors, such as KRAS, BRAF mutation, and microsatellite 
272 instability, which have been proven to be closely related to colon cancer, but because of the

273 limitations of the datasets, we did not perform a stratified analysis on the basis of these factors.

274 Future studies should be conducted to explore the difference under different conditions. Third,

275 the mean time of follow-up in the validation cohort was 65.3 months; thus, a study including a

276 longer follow-up time is warranted to validate our results in the future. Fourth, this study only

277 used a GSE dataset to perform bioinformatics to search for biomarkers. Future studies should 278 implement Partek Genomics software and Illumina Correlation Engine for bioset analyses.

\section{Conclusions}

In this study, we screened gene profiles involved in the metastasis of colon cancer and identified four hub genes from the profiles. We also identified and validated a six-gene signature that can serve as a prognostic indicator of colon cancer. Some genes that have not yet been proven to be associated with colon cancer metastasis may represent new therapeutic targets.

285

286

287 288

289

290

291

292

293

294 295

\section{Abbreviation:}

CRC: colorectal cancer; DEGs: differentially expressed genes; GO: Gene Ontology; KEGG: Kyoto Encyclopedia of Genes and Genomes; OS: overall survival; ROC: receiver operating characteristic curve; OXCT1: 3-oxoacid CoA-transferase 1; ACTN4: actinin alpha 4; IL-8: interleukin 8; ITGA3: integrin subunit alpha 3; ALDH2: aldehyde dehydrogenase 2; NEDD9: neural precursor cell expressed, developmentally down-regulated 9; FLNA: filamin A; LBR: lamin B receptor; TWF1: twinfilin actin binding protein 1; SRSF1: serine and arginine rich splicing factor 1; TNC: tenascin C; RBM47: RNA binding motif protein 47; MET: MET protooncogene, receptor tyrosine kinase 
296

297

298

299

300

301

302

303

304

305

306

307

308

309

310

311

312

313

314

315

316

317

318

\section{Reference}

Castro E, Goh C, Olmos D, Saunders E, Leongamornlert D, Tymrakiewicz M, Mahmud N, Dadaev T, Govindasami K, Guy M, Sawyer E, Wilkinson R, Ardern-Jones A, Ellis S, Frost D, Peock S, Evans DG, Tischkowitz M, Cole T, Davidson R, Eccles D, Brewer C, Douglas F, Porteous ME, Donaldson A, Dorkins H, Izatt L, Cook J, Hodgson S, Kennedy MJ, Side LE, Eason J, Murray A, Antoniou AC, Easton DF, Kote-Jarai Z, and Eeles R. 2013. Germline BRCA mutations are associated with higher risk of nodal involvement, distant metastasis, and poor survival outcomes in prostate cancer. J Clin Oncol 31:17481757. 10.1200/JCO.2012.43.1882

Chen CH, Joshi AU, and Mochly-Rosen D. 2016. The Role of Mitochondrial Aldehyde Dehydrogenase $2(\mathrm{ALDH} 2)$ in Neuropathology and Neurodegeneration. Acta Neurol Taiwan 25(4):111-123. 10196099/254111 [pii]

Dai J, Van Wie PG, Fai LY, Kim D, Wang L, Poyil P, Luo J, and Zhang Z. 2016. Downregulation of NEDD9 by apigenin suppresses migration, invasion, and metastasis of colorectal cancer cells. Toxicol Appl Pharmacol 311:106-112. 10.1016/j.taap.2016.09.016

David JM, Dominguez C, Hamilton DH, and Palena C. 2016. The IL-8/IL-8R Axis: A Double Agent in Tumor Immune Resistance. Vaccines (Basel) 4. 10.3390/vaccines4030022

Deliu IC, Georgescu EF, and Bezna MC. 2014. Analysis of prognostic factors in colorectal carcinoma. Rev Med Chir Soc Med Nat Iasi 118:808-816.

Demirkol S, Gomceli I, Isbilen M, Dayanc BE, Tez M, Bostanci EB, Turhan N, Akoglu M, Ozyerli E, Durdu S, Konu O, Nissan A, Gonen M, and Gure AO. 2017. A Combined ULBP2 and SEMA5A Expression Signature as a Prognostic and Predictive Biomarker 
for Colon Cancer. J Cancer 8:1113-1122. 10.7150/jca.17872

Duband-Goulet I, Courvalin JC, and Buendia B. 1998. LBR, a chromatin and lamin binding protein from the inner nuclear membrane, is proteolyzed at late stages of apoptosis. $J$ Cell Sci 111 ( Pt 10):1441-1451.

Fukumoto M, Kurisu S, Yamada T, and Takenawa T. 2015. alpha-Actinin-4 enhances colorectal cancer cell invasion by suppressing focal adhesion maturation. PLoS One 10:e0120616. 10.1371/journal.pone.0120616

Goncalves V, Henriques AF, Pereira JF, Neves Costa A, Moyer MP, Moita LF, Gama-Carvalho M, Matos P, and Jordan P. 2014. Phosphorylation of SRSF1 by SRPK1 regulates alternative splicing of tumor-related Raclb in colorectal cells. RNA 20:474-482. 10.1261/rna.041376.113

Heagerty PJ, Lumley T, and Pepe MS. 2000. Time-dependent ROC curves for censored survival data and a diagnostic marker. Biometrics 56:337-344.

Hu Y, Chen HY, Yu CY, Xu J, Wang JL, Qian J, Zhang X, and Fang JY. 2014. A long noncoding RNA signature to improve prognosis prediction of colorectal cancer. Oncotarget 5:2230-2242. 1895 10.18632/oncotarget.1895

Hur K, Toiyama Y, Schetter AJ, Okugawa Y, Harris CC, Boland CR, and Goel A. 2015. Identification of a metastasis-specific MicroRNA signature in human colorectal cancer. $J$ Natl Cancer Inst 107. 10.1093/jnci/dju492

Ji H, Greening DW, Barnes TW, Lim JW, Tauro BJ, Rai A, Xu R, Adda C, Mathivanan S, Zhao W, Xue Y, Xu T, Zhu HJ, and Simpson RJ. 2013. Proteome profiling of exosomes derived from human primary and metastatic colorectal cancer cells reveal differential expression of key metastatic factors and signal transduction components. Proteomics 
343

Knijn N, Tol J, and Punt CJ. 2010. Current issues in the targeted therapy of advanced colorectal cancer. Discov Med 9:328-336.

Lambrechts D, Thienpont B, Thuillier V, Sagaert X, Moisse M, Peuteman G, Pericay C, Folprecht G, Zalcberg J, Zilocchi C, Margherini E, Chiron M, and Van Cutsem E. 2015. Evaluation of efficacy and safety markers in a phase II study of metastatic colorectal cancer treated with aflibercept in the first-line setting. Br J Cancer 113:1027-1034. $10.1038 /$ bjc. 2015.329

Lee CL, Huang CJ, Yang SH, Chang CC, Huang CC, Chien CC, and Yang RN. 2016. Discovery of genes from feces correlated with colorectal cancer progression. Oncol Lett 12:33783384. $10.3892 / \mathrm{ol} .2016 .5069$

Li H, Chen YX, Wen JG, and Zhou HH. 2017. Metastasis-associated in colon cancer 1: A promising biomarker for the metastasis and prognosis of colorectal cancer. Oncol Lett 14:3899-3908. 10.3892/ol.2017.6670

Li R, Zhao Z, Sun M, Luo J, and Xiao Y. 2016. ALDH2 gene polymorphism in different types of cancers and its clinical significance. Life Sci 147:59-66. 10.1016/j.lfs.2016.01.028

Liew AW, Law NF, and Yan H. 2011. Missing value imputation for gene expression data: computational techniques to recover missing data from available information. Brief Bioinform 12:498-513. 10.1093/bib/bbq080

Linhares MM, Affonso RJ, Jr., Viana Lde S, Silva SR, Denadai MV, de Toledo SR, and Matos D. 2015. Genetic and Immunohistochemical Expression of Integrins ITGAV, ITGA6, and ITGA3 As Prognostic Factor for Colorectal Cancer: Models for Global and Disease-Free Survival. PLoS One 10:e0144333. 10.1371/journal.pone.0144333 
365 Marzouk O, and Schofield J. 2011. Review of histopathological and molecular prognostic 366 features in colorectal cancer. Cancers (Basel) 3:2767-2810. 10.3390/cancers3022767

Nagata M, Noman AA, Suzuki K, Kurita H, Ohnishi M, Ohyama T, Kitamura N, Kobayashi T, Uematsu K, Takahashi K, Kodama N, Kawase T, Hoshina H, Ikeda N, Shingaki S, and Takagi R. 2013. ITGA3 and ITGB4 expression biomarkers estimate the risks of locoregional and hematogenous dissemination of oral squamous cell carcinoma. $B M C$ Cancer 13:410. 10.1186/1471-2407-13-410

Paavilainen VO, Hellman M, Helfer E, Bovellan M, Annila A, Carlier MF, Permi P, and Lappalainen P. 2007. Structural basis and evolutionary origin of actin filament capping by twinfilin. Proc Natl Acad Sci U S A 104:3113-3118. 10.1073/pnas.0608725104

Provenzani A, Fronza R, Loreni F, Pascale A, Amadio M, and Quattrone A. 2006. Global alterations in mRNA polysomal recruitment in a cell model of colorectal cancer progression to metastasis. Carcinogenesis 27:1323-1333. 10.1093/carcin/bgi377

Qin S, Kim J, Arafat D, and Gibson G. 2012. Effect of normalization on statistical and biological interpretation of gene expression profiles. Front Genet 3:160. 10.3389/fgene.2012.00160

Roessler S, Lin G, Forgues M, Budhu A, Hoover S, Simpson RM, Wu X, He P, Qin LX, Tang $\mathrm{ZY}$, Ye QH, and Wang XW. 2015. Integrative genomic and transcriptomic characterization of matched primary and metastatic liver and colorectal carcinoma. Int $J$ Biol Sci 11:88-98. 10.7150/ijbs.10583

Rokavec M, Kaller M, Horst D, and Hermeking H. 2017. Pan-cancer EMT-signature identifies RBM47 down-regulation during colorectal cancer progression. Sci Rep 7:4687. $10.1038 / \mathrm{s} 41598-017-04234-2$

Sameer AS. 2013. Colorectal cancer: molecular mutations and polymorphisms. Front Oncol 
388

389

390

391

392

393

394

395

396

397

398

399

400

401

402

403

404

405

406

407

408

409

410

3:114. 10.3389/fonc. 2013.00114

Sanford JR, Wang X, Mort M, Vanduyn N, Cooper DN, Mooney SD, Edenberg HJ, and Liu Y. 2009. Splicing factor SFRS1 recognizes a functionally diverse landscape of RNA transcripts. Genome Res 19:381-394. 10.1101/gr.082503.108

Shagisultanova E, Gaponova AV, Gabbasov R, Nicolas E, and Golemis EA. 2015. Preclinical and clinical studies of the NEDD9 scaffold protein in cancer and other diseases. Gene 567:1-11. 10.1016/j.gene.2015.04.086

Sheffer M, Bacolod MD, Zuk O, Giardina SF, Pincas H, Barany F, Paty PB, Gerald WL, Notterman DA, and Domany E. 2009. Association of survival and disease progression with chromosomal instability: a genomic exploration of colorectal cancer. Proc Natl Acad Sci U S A 106:7131-7136. 10.1073/pnas.0902232106

Shelmerdine SC, Semple T, Wallis C, Aurora P, Moledina S, Ashworth MT, and Owens CM. 2017. Filamin A (FLNA) mutation-A newcomer to the childhood interstitial lung disease (ChILD) classification. Pediatr Pulmonol 52:1306-1315. 10.1002/ppul.23695

Siegel RL, Miller KD, Fedewa SA, Ahnen DJ, Meester RGS, Barzi A, and Jemal A. 2017a. Colorectal cancer statistics, 2017. CA Cancer J Clin 67:177-193. 10.3322/caac.21395

Siegel RL, Miller KD, and Jemal A. 2017b. Cancer Statistics, 2017. CA Cancer J Clin 67:7-30. $10.3322 /$ caac. 21387

Stein U, and Schlag PM. 2007. Clinical, biological, and molecular aspects of metastasis in colorectal cancer. Recent Results Cancer Res 176:61-80.

Tian ZQ, Shi JW, Wang XR, Li Z, and Wang GY. 2015. New cancer suppressor gene for colorectal adenocarcinoma: filamin A. World $J$ Gastroenterol 21:2199-2205. 10.3748/wjg.v21.i7.2199 
411 Vellinga TT, Kranenburg O, Frenkel N, Ubink I, Marvin D, Govaert K, van Schelven S, 412 Hagendoorn J, and Borel Rinkes IH. 2017. Lymphangiogenic Gene Expression Is Associated With Lymph Node Recurrence and Poor Prognosis After Partial Hepatectomy

414 for Colorectal Liver Metastasis. Ann Surg. 10.1097/SLA.0000000000002430

$415 \mathrm{Xu} \mathrm{G}$, Zhang M, Zhu H, and Xu J. 2017. A 15-gene signature for prediction of colon cancer 416 recurrence and prognosis based on SVM. Gene 604:33-40. 10.1016/j.gene.2016.12.016

417 Zarour LR, Anand S, Billingsley KG, Bisson WH, Cercek A, Clarke MF, Coussens LM, Gast CE, 418 Geltzeiler CB, Hansen L, Kelley KA, Lopez CD, Rana SR, Ruhl R, Tsikitis VL, Vaccaro 419 GM, Wong MH, and Mayo SC. 2017. Colorectal Cancer Liver Metastasis: Evolving 420 Paradigms and Future Directions. Cell Mol Gastroenterol Hepatol 3:163-173. $421 \quad$ 10.1016/j.jcmgh.2017.01.006

422 Zhang S, and Xie C. 2017. The role of OXCT1 in the pathogenesis of cancer as a rate-limiting 423 enzyme of ketone body metabolism. Life Sci 183:110-115. 10.1016/j.lfs.2017.07.003 
Figure 1

Venn diagrams showing intersected genes overlapping between time-series genes (TSGs) and differentially expressed genes (DEGs).

\section{GSE2509}

GSE40367

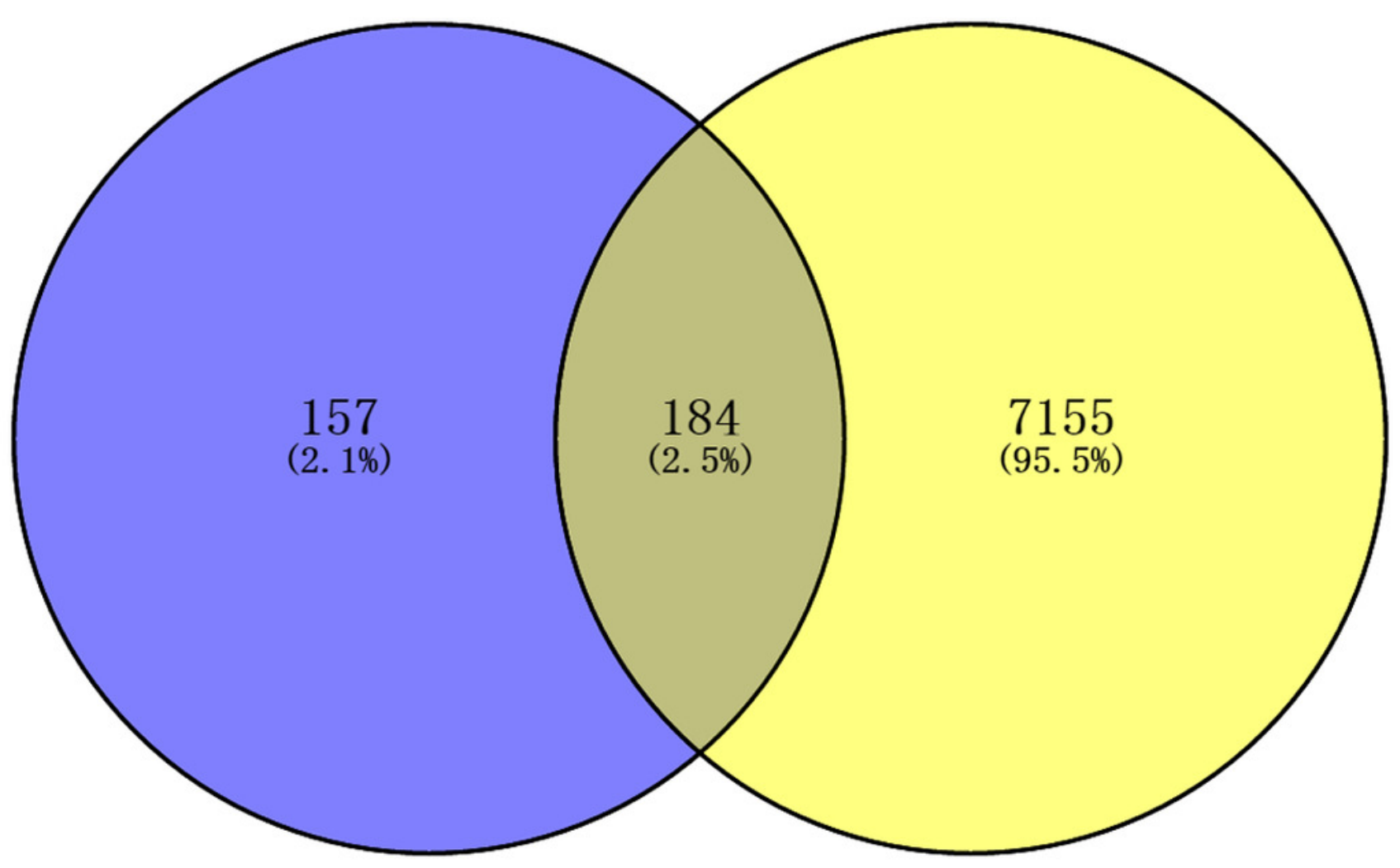


Figure 2

Functional enrichment analysis of overlapped DEGs.

A signal transduction

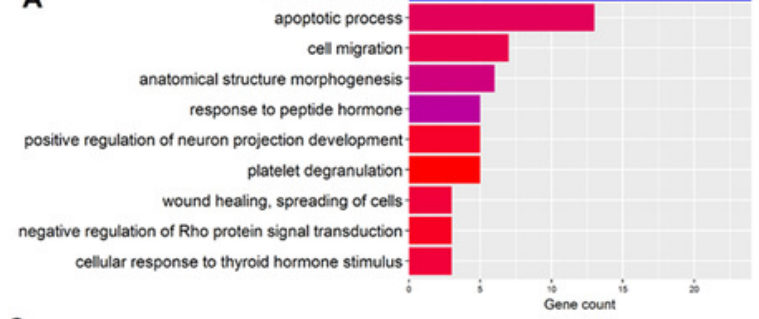

C

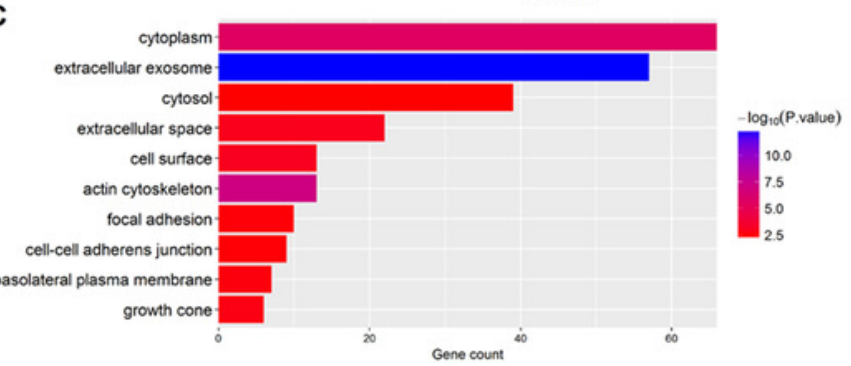

B

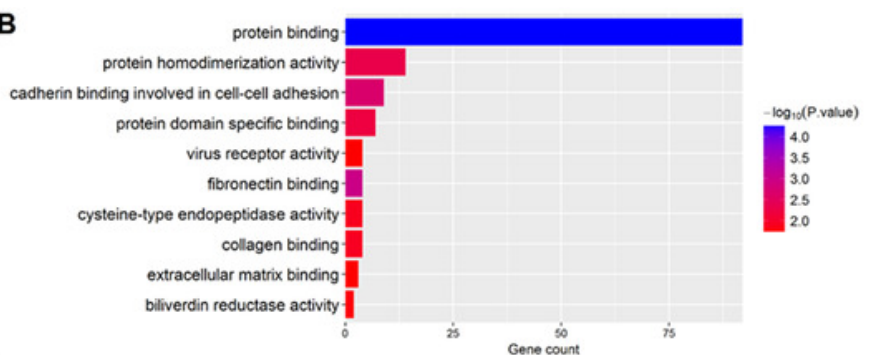

D

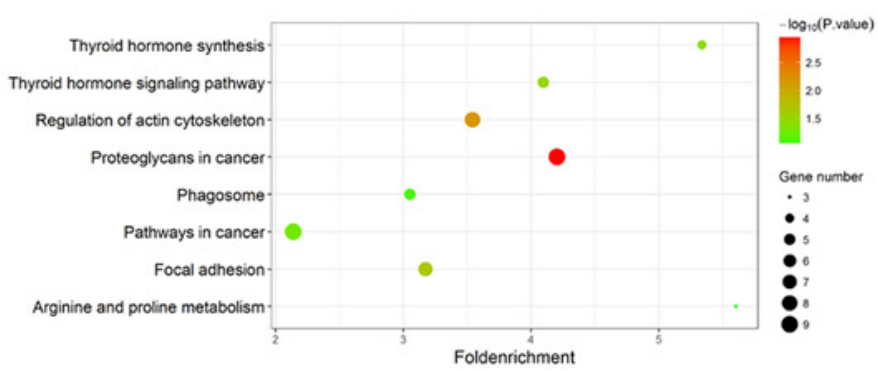


Figure 3

The protein-protein interaction (PPI) network analysis of the 326 overlapped genes.
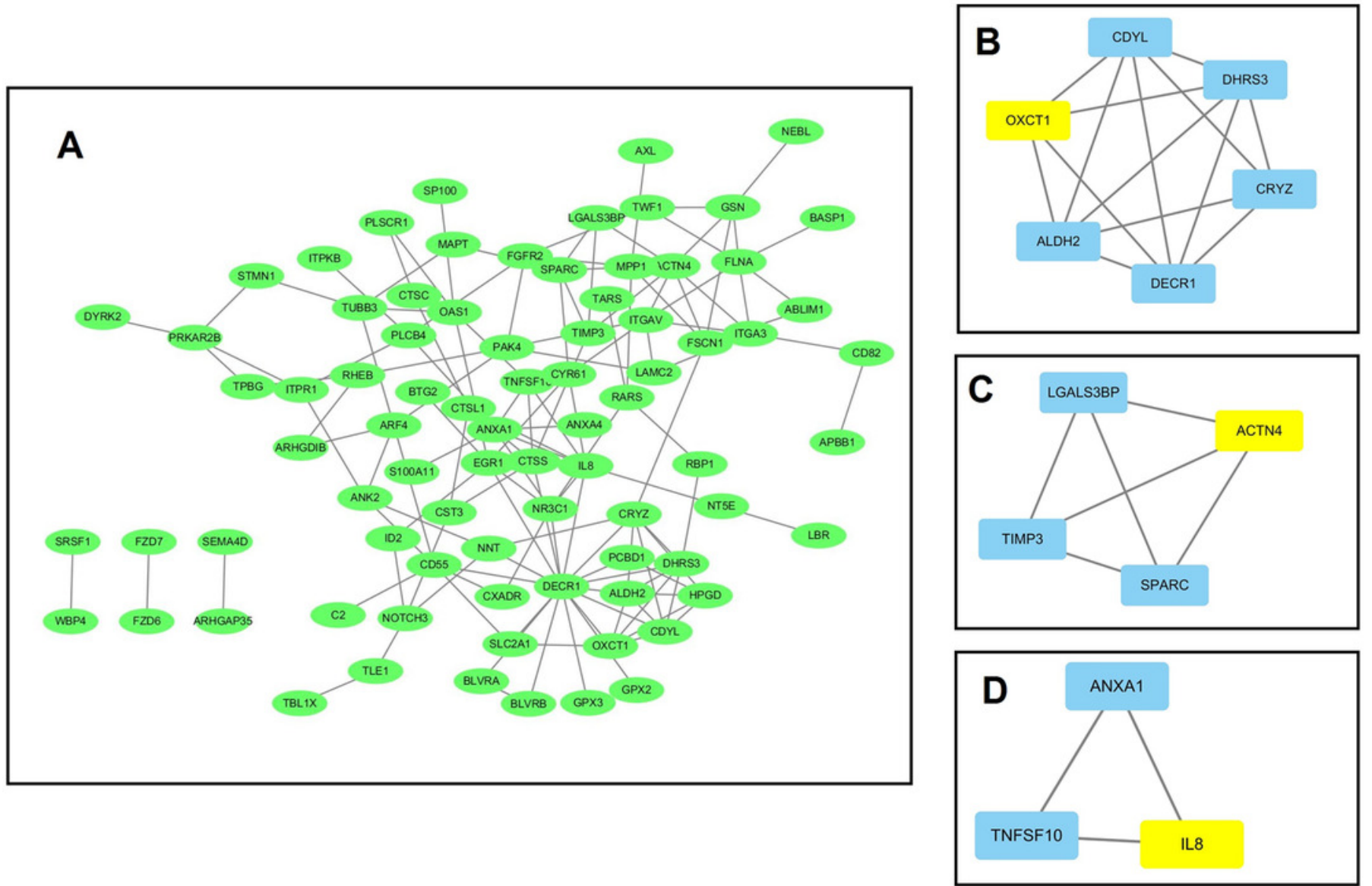


\section{Figure 4}

The prognostic performance of the six-gene signature of colon cancer.

A: Risk score curve of the six-gene signature; B: Patient survival status and time distribution by risk score; Heatmap showed the expression of six genes by risk score. 
A

B
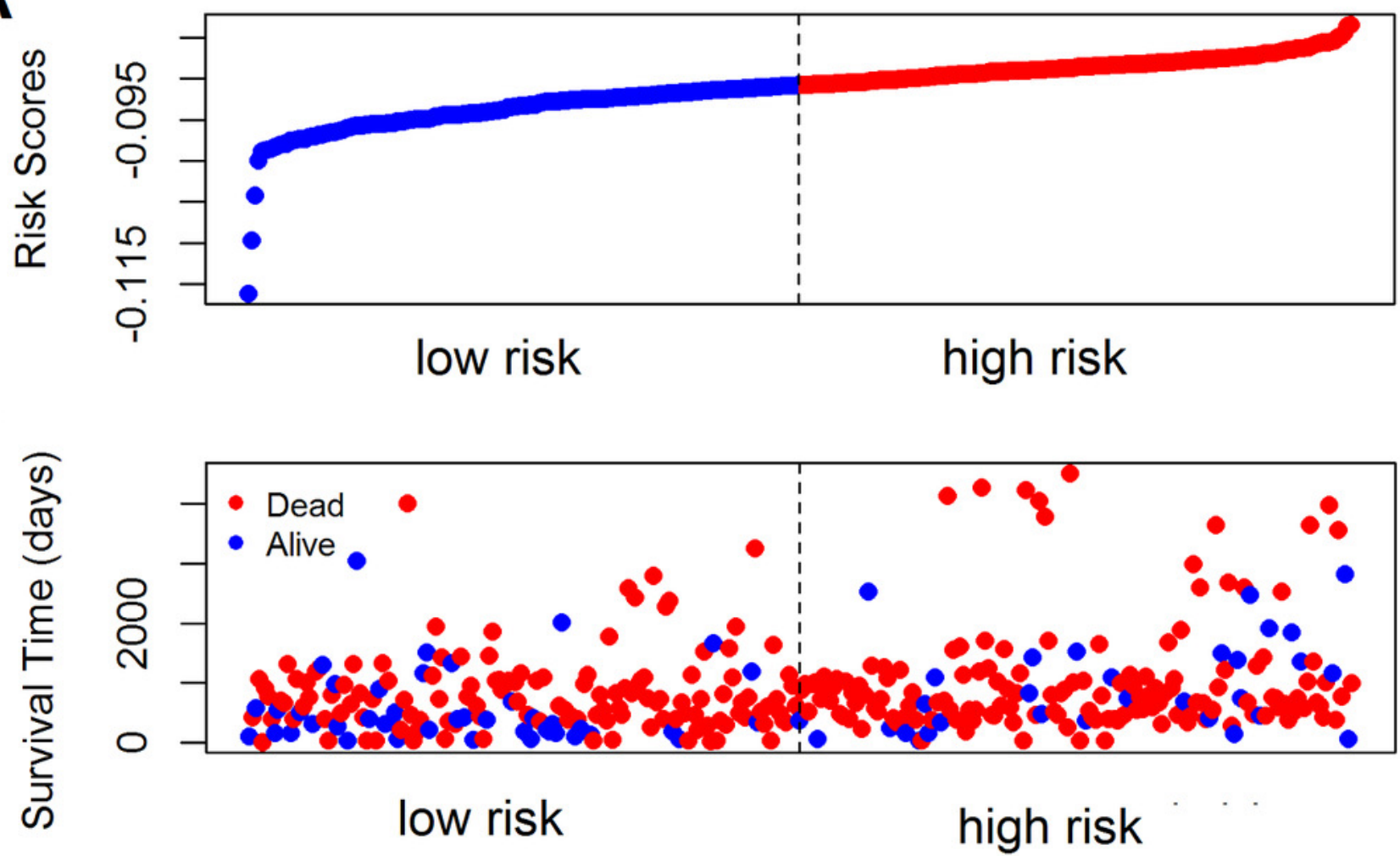

C

SFRS1

$\mathrm{ALDH} 2$

TWF1

LBR

FLNA

NEDD9

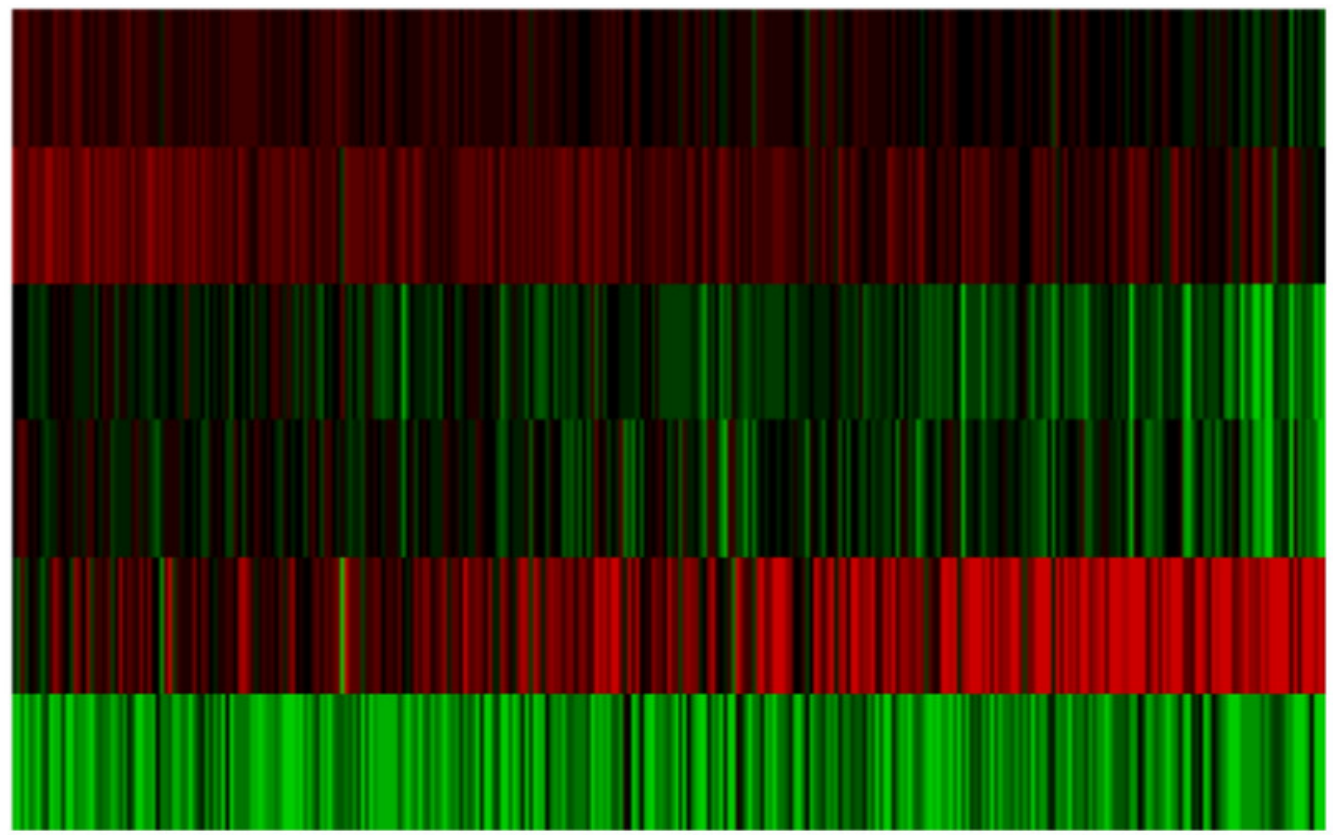


Figure 5

Survival analysis for the gene signature

A: The Kaplan-Meier test of the risk score for the overall survival of colon cancer between high-risk and low-risk patients; B: The prognostic value of the risk score showed by the timedependent receiver operating characteristic (ROC) curve for predicting the 1, 3, 5- years overall survival.
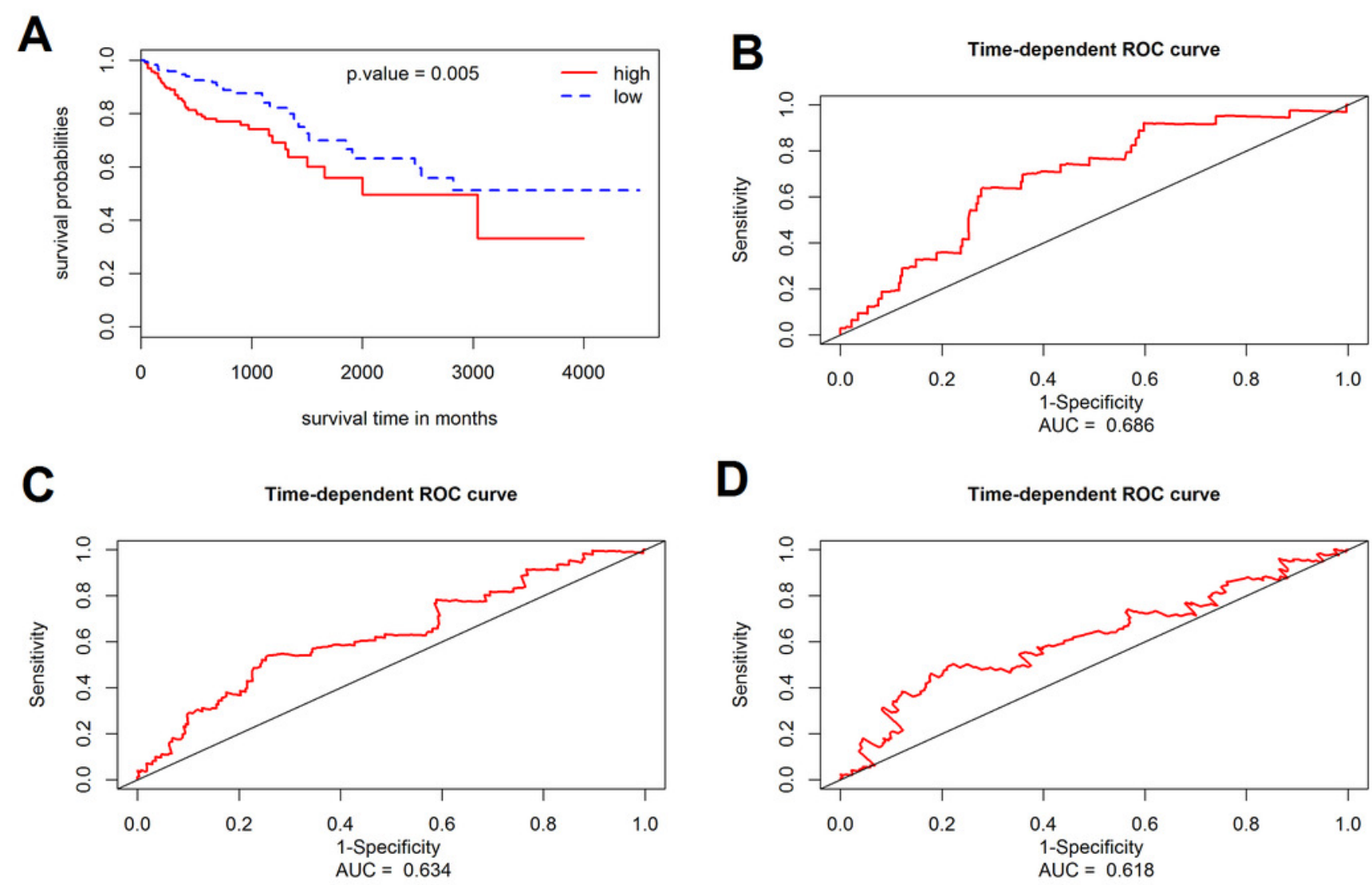

D

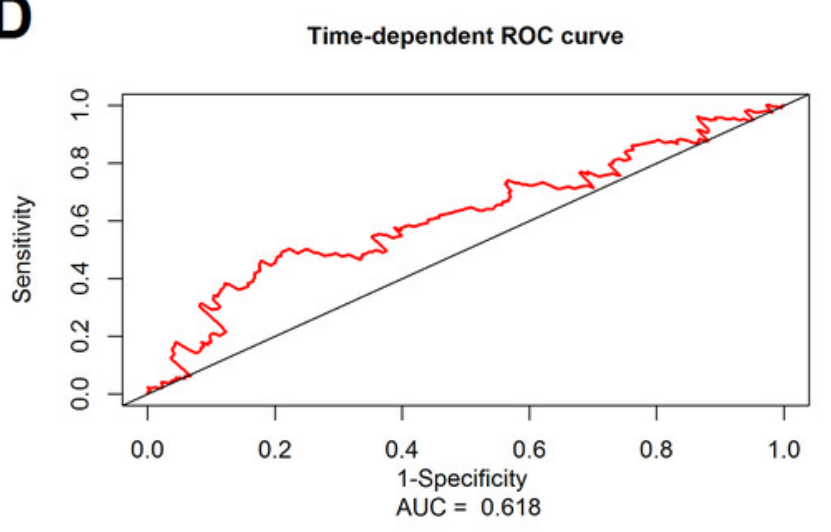

